\title{
An overview of recent site survey studies conducted for ELT projects: aerial surveys and site comparisons of cloud cover and water vapour using satellite data
}

\author{
D. André Erasmus \\ South African Astronomical Observatory, P.O. Box 9, Observatory, 7935, South Africa \\ email: erasmus@saao.ac.za
}

\begin{abstract}
Areas surveyed for ELT projects using satellite data are described. A synopsis of the methodology used is given and selected results from recent studies are presented.
\end{abstract}

Keywords. Site survey studies, satellite, cloud cover, water vapour

\section{Introduction}

Over the last five years a number of site survey studies have been carried out for ELT projects to survey cloud cover and precipitable water vapour (PWV) at candidate telescope sites using meteorological satellite data. The aerial distribution of cloud cover and PWV was mapped. Then the best sites were identified, analysed in further detail, compared and ranked.

Three studies have been conducted for what is now known as the Thirty Meter Telescope (TMT) site survey. Cloud cover and PWV in Northern Chile over the area $20.5^{\circ} \mathrm{S}$ to $30.5^{\circ} \mathrm{S}$ and $66.0^{\circ} \mathrm{W}$ to $72.0^{\circ} \mathrm{W}$ were analysed. Based on the results of the aerial analysis, the initial list of 37 existing and potential telescope sites was reduced to 14 . Conditions at these sites were compared and the sites were ranked in terms of their observing quality. Later the area $18^{\circ} \mathrm{N}$ to $40^{\circ} \mathrm{N}$ and $96^{\circ} \mathrm{W}$ to $124^{\circ} \mathrm{W}$ over the Southwest USA and Northern Mexico was surveyed. From an original list of 33 sites the 10 best potential telescope sites and 5 pre-selected sites were identified. The short list included existing observatories with ground-based measurements of cloud and PWV. These were used to validate the satellite observations. Using the results from the first two studies nine sites in Northern Chile and three sites in the North American study area were compared to Mauna Kea, Hawaii. For these studies 58 months of satellite data from the Geostationary Operational Environmental Satellites (GOES) positioned at $75^{\circ} \mathrm{W}$ and $135^{\circ} \mathrm{W}$ were used. These same data were also used to compare sites in Chile, Argentina and Bolivia for the Atacama Large Millimeter Array (ALMA) project.

Two sites in the Himalayan region (Hanle, India: $32.78^{\circ} \mathrm{N}, 78.96^{\circ} \mathrm{E}$ and Yanbajing, Tibet: $30.11^{\circ} \mathrm{N}, 90.53^{\circ} \mathrm{E}$ ) have also been compared. This area was being considered for next-generation Imaging Atmospheric Cherenkov Telescopes. The satellite data used in this study were from the Meteosat Indian Ocean Data Coverage Service located in geostationary orbit at $63^{\circ} \mathrm{E}$ for the period 1 July, 1998 to 30 June, 2003.

An area covering Northwest Africa between $20^{\circ} \mathrm{N}$ to $40^{\circ} \mathrm{N}$ and $20^{\circ} \mathrm{W}$ to $10^{\circ} \mathrm{E}$ is currently being surveyed for the Overwhelmingly Large (OWL) telescope project. The satellite data being used in this study are from the Meteosat Operational Service in geostationary orbit at $0^{\circ}$ longitude and covers the 7-year period January 1996 to December 2002 . 


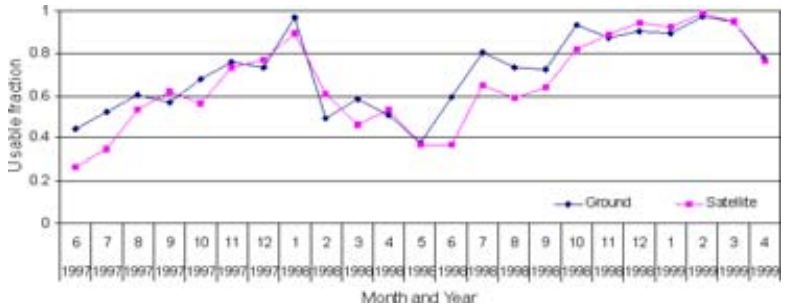

Figure 1. Monthly usable (photometric plus spectroscopic) fraction at Cerro Tololo Inter-American Observatory as determined from satellite and ground-based observations of cloud cover over the period June 1997 to April 1999.

\section{Methodology}

In the studies mentioned above measurements of cloud cover and PWV are derived from satellite observations made by passive remote sensing at $\pm 11 \mu \mathrm{m}$ and $\pm 6.5 \mu \mathrm{m}$. Satellite observations at about $6.5 \mu \mathrm{m}$ are sensitive to emissions from WV resident in the layer between about $600 \mathrm{mb}( \pm 4400 \mathrm{~m})$ and $300 \mathrm{mb}( \pm 9000 \mathrm{~m})$. If high altitude (cirrus) clouds exist above this layer their presence and thickness can be determined. The other channel is used to detect clouds at middle and low levels.

Analysis can be performed for a specific site or an area of interest. Rather than using just one pixel to represent the "sky" above a particular location, a more realistic picture is obtained using a $9(3 \times 3)$-pixel area. For the area analysis the 9 -pixel template is passed over the area of interest, counts compiled and contours drawn. For a detailed description of the methodology employed in the above mentioned studies please consult Erasmus \& Sarazin (2000), Erasmus \& Sarazin (2002), Erasmus (2005).

\section{Results}

Within the scope of this paper it is only possible to present a small sample of the results obtained in the surveys conducted to date. The two main types of analyses that have been performed are for individual sites, site comparisons and for areas of interest. Examples of each are presented below. Since some of the results, particularly those comparing specific sites, continue to be client privileged these could not be included (for more information contact the author).

Figure 1 shows the monthly usable (photometric plus spectroscopic) fraction at Cerro Tololo Observatory as determined from satellite and ground-based observations of cloud cover over the period June 1997 to April 1999 (Erasmus \& Van Staden 2003). This

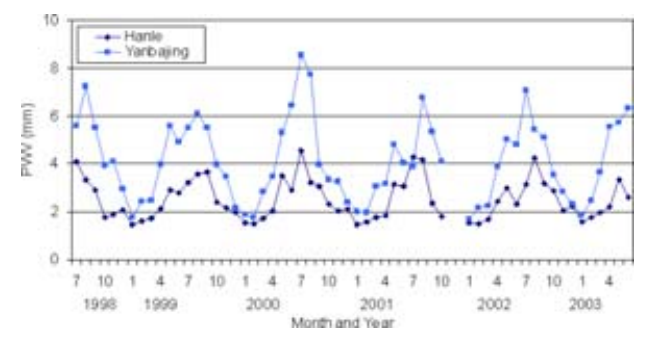

Figure 2. Monthly PWV for Hanle, India and Yanbajing, China over the period July 1998 to April 2003.

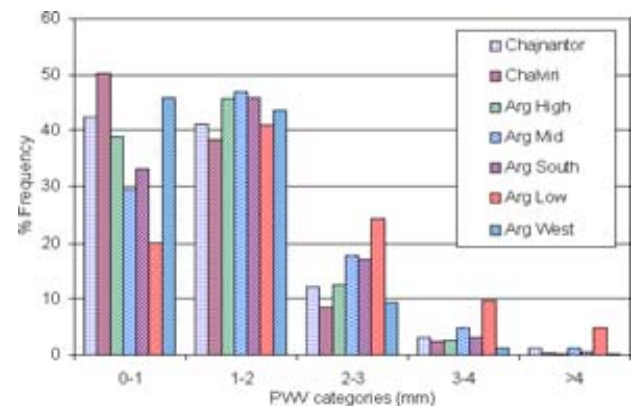

Figure 3. Histogram comparing PWV at Chajnantor (Chile), Chalviri (Bolivia) and four sites in Argentina using 60 months of data between July 1993 and September 1999. 

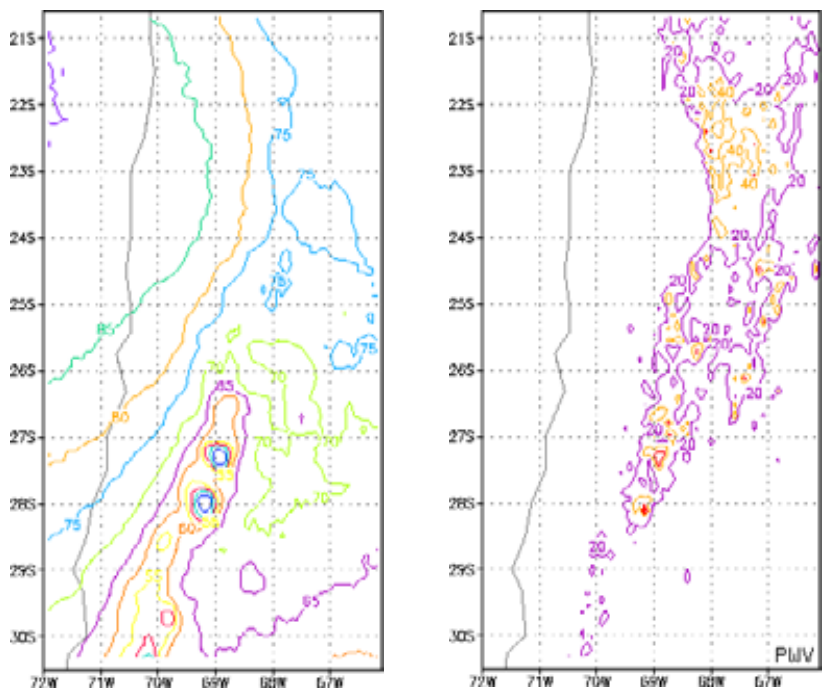

Figure 4. Maps showing the aerial distribution of cloud cover for the observing night and PWV over Northern Chile. Isokephs show the percentage of time that skies are clear over the study period. The second map (marked PWV) shows the percentage frequency of occurrence of PWV values less than $1 \mathrm{~mm}$ for the atmospheric column above the $700 \mathrm{mb}$ pressure level $( \pm 3300 \mathrm{~m})$ or the surface (if higher).

comparison shows that the satellite "sees" the cloud cover in a similar manner to a ground-based observer. In the case of PWV good agreement between the satellite and ground-based measurements has also been observed at several sites (Erasmus 2002).

Figure 2 shows the mean monthly PWV for Hanle, India, and Yanbajing, Tibet, over the period July 1998 to April 2001 (Erasmus 2004). The seasonal dependence in PWV is readily seen. Figure 3 shows histograms comparing PWV at high altitude sites in Argentina, Chile and Bolivia.

The maps that follow show the aerial distribution of cloud cover and/or PWV over three of the study areas described above. The first of these (Figure 4) is for Northern Chile (Erasmus \& Van Staden 2001). The optimal latitude belt for clear skies over Northern Chile is between $22^{\circ} \mathrm{S}$ and $24^{\circ} \mathrm{S}$ with an increase in cloud cover observed towards the East and South. The PWV map shows the superior dryness of high altitude locations.

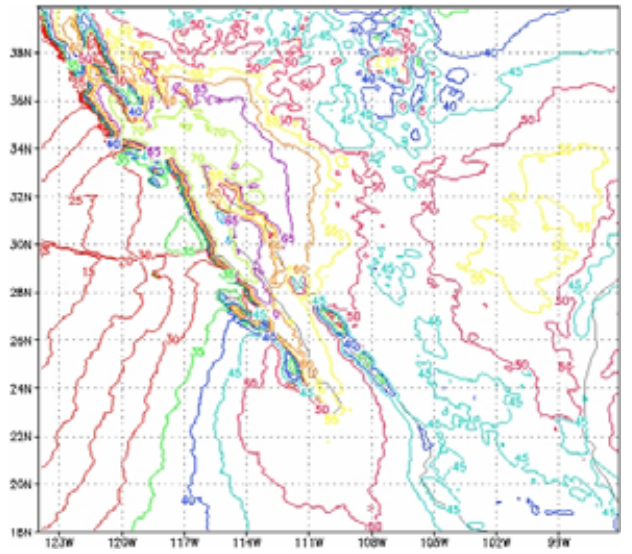

Figure 5. Map showing the clear fraction for the observing night over the Southwest USA and Northern Mexico. Isokephs show the percentage of time that skies are clear over the study period.

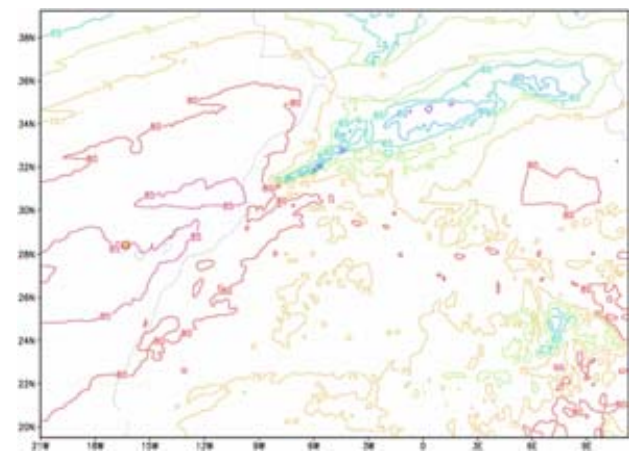

Figure 6. Map showing the clear fraction for the observing night over Northwest Africa and Southern Spain. Isokephs show the percentage of time that skies are clear over the study period. 
Figure 5 shows the fraction of time that skies are clear over the area surveyed in North America (Erasmus \& Van Staden 2002). For the observing night, a large area with a clear fraction of $70 \%$ or more is observed over Southern California, Western Arizona and Northern Baja, Mexico.

The clear fraction for the area surveyed in NW Africa and Southern Spain is shown in Figure 6 (Erasmus \& Van Rooyen 2006). Similar to Northern Chile, the clearest area is observed along the West coast, however, in this area the optimal latitude belt is found further poleward between $25^{\circ}$ and $30^{\circ}$ latitude. There is a marked increase in cloudiness over the Atlas mountains due to orographic lifting. Over the continent therefore, the clearest locations are in the far Southwest of the Anti-Atlas range.

\section{Acknowledgements}

Studies were funded by: Association of Universities for Research in Astronomy, University of Tokyo, European Southern Observatory, University of California, Caltech and Max Planck Institute. The author wishes to thank the IAU for their support to attend the IAU Symposium 232 in Cape Town and acknowledges the following persons for their contributions to the work presented in this paper: Andries van Staden, Ruby van Rooyen and Deneys Maartens.

\section{References}

Erasmus, D.A. \& Sarazin, M. 2000, Forecasting Precipitable Water Vapor and Cirrus Cloud Cover For Astronomical Observatories: Satellite image processing guided by synoptic model dissemination data. SPIE Conference on Image and Signal Processing for Remote Sensing IV. Paper SPIE-4168, Barcelona. 25-29 September, 2000,

Erasmus, D.A. \& Sarazin, M. 2002, in: J. Vernin, Z. Benkhaldoun, C. Muñoz-Tuñón (eds.), Astronomical Site Evaluation in the Visible and Radio Range, (San Franciso: ASP) 266, 310

Erasmus, D.A. 2005, www.astro.caltech.edu/ erasmus

Erasmus, D.A. \& Van Staden, C.A. 2003, A Comparison of Satellite-Observed Cloud Cover and Water Vapor at Mauna Kea and Selected Sites in Northern Chile, the Southwest USA and Northern Mexico. Final Report. 31 August, 2003. New Initiatives Office, Aura Inc.

Erasmus, D.A. 2002, An Analysis of Cloud Cover and Water Vapor for the ALMA Project: A Comparison Between Chajnantor (Chile), Chalviri (Bolivia) and Five Sites in Argentina using Satellite Data and a Verification of Satellite PWV measurements. (www.eso.org/ gen-fac/pubs/astclim/espas/radioseeing/)

Erasmus, D.A. 2004, An Analysis and Comparison Of Satellite-Observed Cloud Cover and Water Vapor At Hanle, India and Yanbajing, Tibet. Experimental Particle Physics and High Energy Astrophysics Group. The Max-Planck-Institute for Nuclear Physics Heidelberg. Final Report. 31 January, 2004.

Erasmus, D.A. \& Van Staden, C.A. 2001, A Satellite Survey of Water Vapor and Cloud Cover in Northern Chile. Final Report. Cerro-Tololo Inter-American Observatory.

Erasmus, D.A. \& Van Staden, C.A. 2002, A Satellite Survey of Water Vapor and Cloud Cover in the Southwest USA and Northern Mexico. Final Report. Aura Inc and the CELT Project.

Erasmus, D.A. \& Van Rooyen, R. 2006, A Satellite Survey of Cloud Cover and Water Vapour in Morocco and Southern Spain and a Verification Using La Palma Ground-based Observations. Final Report. ESO, In Preparation. 